\title{
Micellar Effects of Cetyl Pyridinium Chloride on Antioxidant Capacity, Voltammetric Response of Serum and Saliva Samples
}

Madla Adami dos Passos ${ }^{1}$, Denise Vaz de Macedo ${ }^{*}$, Rita de Cássia Silva Luz ${ }^{3}$, Lázaro Alessandro Soares Nunes ${ }^{2}$, Lauro Tatsuo Kubota and Armindo Antonio Alves ${ }^{2,5}$

${ }^{1}$ Physical Education Faculty, Campinas State University, P.O. Box, 6134, 13083-970, Campinas, SP, Brazil

${ }^{2}$ Biochemistry Department, Biology Institute, Campinas State University, P.O. Box 6109, 13084-971 Campinas, SP, Brazil

${ }^{3}$ Faculty of Exact Science and Social Applied Science, Jequitinhonha and Mucuri Valleys Federal University, P.O. Box 39100-000, Diamantina, MG, Brazil

${ }^{4}$ Analytical Chemistry Department, Chemistry Institute, Campinas State University, P.O. Box 6154, 13084-971 Campinas, SP, Brazil

${ }^{5}$ Fundação Herminio Ometto-NUCISA, P.O. Box 13607-339, Araras, SP, Brazil

\begin{abstract}
The production of reactive oxygen species due to increased energy demand during physical exercise can increase the cellular and blood oxidation state. The reducing power of biological samples reflects its antioxidant capacity, largely maintained by Low Molecular Weight Antioxidants (LMWA), which donate electrons to radical species. LMWA include antioxidants such as uric acid, vitamins $\mathrm{C}$ and $\mathrm{E}$ and lipoic acid, among others. The electroanalytical technique of Differential Pulse Voltammetry (DPV) presents a good methodological alternative to quantify acute and chronic modulations of the antioxidant capacity from biological fluids in response to metabolic adaptations caused by physical exercise. However, when biological samples are analyzed, proteins are an important preanalytical interfering in the technique. The proteins can be adsorbed on the electrode surface during the potential application, resulting in a significant decrease of voltammetric signal. The aim of the present study was to investigate the applicability of cationic surfactant Cetyl Pyridinium Chloride (CPC) as a micellar system for the improvement of DPV technique for serum and saliva analysis. Forty individuals' samples were analyzed. The obtained data revealed that the use of CPC increased the sensitivity and stability of the voltammetric signal, enabling the application of the method DPV for serum and saliva samples. Our data suggest that the voltammetric signal of samples is influenced mainly by the uric acid concentration.
\end{abstract}

Keywords: Differential pulse voltammetry; Total antioxidant capacity; Low molecular weight antioxidants; Cetyl pyridinium chloride; Serum; Saliva

\section{Introduction}

The quantification of antioxidants has become a topic of increasing interest in interdisciplinary research works involving pathologies and physical exercise [1-5]. Antioxidants can be defined as molecules that can prevent or reduce the extent of oxidative destruction of biomolecules when present in small concentrations compared to those biomolecules $[1,6,7]$.

The antioxidants can be classified in two groups: enzymatic and non-enzymatic or low molecular weight antioxidants (LMWA). The enzymatic antioxidants include a relatively small number of specific enzymes such as superoxide dismutase, catalase and the system of glutathione peroxidase/glutathione reductase. LMWA include many compounds, which can be considered mainly responsible for total antioxidant capacity of tissues and biological fluids [8,9]. LMWA are located within ROS targets and specific sites of ROS generation in the cells. They interact directly with ROS (radical scavengers) and indirectly, chelating metals ions involved in ROS formation. LMWA, synthesized by the cells, can originate from the diet or can be derived from waste products $[9,10]$.

The LMWA that react directly with ROS are reducing agents that donate electrons to radical species. In other words, the antioxidant capacity indicates the reducing power of biological samples. LMWA have electroactivity; therefore, they can be measured by electrochemical methods $[1,10]$.

Differential pulse voltammetry (DPV) and cyclic voltammetry (CV) are widely used methods in analytical chemistry. Both were developed under the same principle, that is, to evaluate the capacity of a sample to donate or to receive electrons from a work electrode that is subjected to a defined potential interval. The voltammetric signal is formed by two parameters: the peak potential, which is determined by the redox potential of the analyzed compounds, and the peak current, which is a direct function of its concentration $[11,12]$.

Validity, sensitivity and reproducibility are three factors that need to be considered to select and to define an analysis method [13]. Validity is the capacity of a measurement tool to reflect what it is designed to measure, in this case, the antioxidant capacity of serum and saliva given by the reducing power of LMWA. The sensitivity is the capacity of the technique to detect small alterations. The reproducibility of an analysis method consists of the absence of systematic and random error in repeated trials $[14,15]$.

Chevion et al. [8] developed a procedure using $\mathrm{CV}$ to determine the antioxidant capacity of plasma samples. However, the DPV method has several advantages compared to $\mathrm{CV}$ such as good discrimination against background currents, better sensitivity and lower detection limits [12].

The large limitation of the applicability of electroanalytical

${ }^{*}$ Corresponding author: Armindo Antonio Alves, Biochemistry Department Biology Institute, Campinas State University, Brazil, Tel: 55-19-96270855; Fax: 5519-3521-6129; E-mail: alvesaa@uol.com.br

Received April 24, 2012; Accepted November 05, 2012; Published November 07, 2012

Citation: de Passos MA, de Macedo DV, de Cássia Silva Luz R, Nunes LAS Kubota4 LT, et al. (2012) Micellar Effects of Cetyl Pyridinium Chloride on Antioxidant Capacity, Voltammetric Response of Serum and Saliva Samples. J Biosens Bioelectron 3:130. doi:10.4172/2155-6210.1000130

Copyright: (C) 2012 de Passos MA, et al. This is an open-access article distributed under the terms of the Creative Commons Attribution License, which permits unrestricted use, distribution, and reproduction in any medium, provided the original author and source are credited. 
methods is the gradual passivation of the working electrode by the species generated in the electrochemical reaction [16]. Bare electrodes often suffer from a fouling effect due to the accumulation of oxidized products on their surfaces. All these result in low selectivity and sensitivity [17]. When we consider biological samples, the proteins are significant interfering agents. They can be adsorbed on the electrode surface during the application of potential and result in a decrease or even a complete loss of the signal during repeated measurements with the same electrode [18]. The passivation can be avoided through the polishing of the working electrode before each analysis [10]. However, our preliminary data showed that the polishing alters the electrode surface, constituting a limitation in the application of the technique of DPV to analyze the reducing power of biological samples.

Several strategies have been developed for overcoming the adsorption interferences, including mineralization of the sample [19], coating of the working electrode with a permselective membrane [20], microwave activation and ultrasonication [21]. These techniques involve different analytical procedures and complex instrumentation [22]. Therefore, it is necessary to develop new techniques that enable the use of biological samples in electrochemical analyses.

To that end, the use of surfactants for the stabilization of voltammetric signal is a simple alternative. The sole required manipulations are the addition of the surfactant and the thermostatting of the electrochemical cell. Micellar aggregates assembled from surfactants can be adsorbed on electrodes and may mediate catalytic systems $[23,24]$.

Surfactants have also been used as selective masking agents to improve selectivity and sensitivity of electrochemical analysis [23,25]. Hoyer and Jensen [16] showed that the fall-off of voltammetric serotonin signal could be reduced by surfactant addition in the sample. The stabilization effect of surfactants could be attributed to the competitive adsorption at the electrode surface, which counteracts the accumulation of oxidation products [18]

The behavior of the electrochemistry oxidation of ascorbic acid in aqueous solution with different surfactants has been studied by Szymula and Nazkiewickz-Michalek [26]. It was verified that the cationic surfactant shifts the oxidation peak potential and changes the peak current, mainly due to the surfactant film formed at the electrode/ solution interface. The negative charge of ascorbic acid interacts with the positive charge of cationic micelles, which enhances the rate of oxidation and consequently increases the peak current. The use of nonionic and anionic surfactants acted in the opposite way.

Thus, the present work describes a study based on differential pulse voltammetry (DPV) techniques for determination of antioxidant capacity in serum and saliva samples in a cationic micellar system (cetyl pyridinium chloride (CPC)) using a glassy carbon electrode as the working electrode. The other goal was to validate the VPD in a cationic micellar system CPC as a useful method for direct measurement of antioxidant capacity conferred by ABPM serum and saliva.

\section{Materials and Methods}

\section{Reagents and solutions}

All reagents were of analytical grade and were used as received without further purification. Cetyl pyridinium chloride monohydrate $\left(\mathrm{C}_{21} \mathrm{H}_{38} \mathrm{NCl} \bullet \mathrm{H}_{2} \mathrm{O}\right), \mathrm{NaCl}$, uric acid and ascorbic acid were acquired from Sigma Aldrich. Disodium and monosodium phosphates $\left(\mathrm{Na}_{2} \mathrm{HPO}_{4}\right.$ and $\mathrm{NaH}_{2} \mathrm{PO}_{4}$ ) were acquired from Merck (KgaA Germany).

The solutions of cetyl pyridinium chloride monohydrate, uric acid, ascorbic acid and PBS (aqueous phosphate buffer $0.5 \mathrm{molL}^{-1}, 0.9 \%$ of $\mathrm{NaCl}, \mathrm{pH}=7.4$ ) were prepared using water purified by a millipore milli-Q system $\left(\mathrm{p}>18.2 \mathrm{M}^{\prime} \Omega\right)$.

\section{Serum samples and saliva}

Blood and saliva samples were collected from forty volunteer soccer players (18 \pm 1 years old). This study was approved by Committee of Ethics in Human Research (State University of Campinas) (0200.0.146.000-08).

In the morning, $8.0 \mathrm{~mL}$ of blood were collected in Vacuette tubes (Greiner Bio-One GmbH, Kremsmuenter, Austria). The volunteers fasted for at least 8 hours. The collected blood was centrifuged at 2000 $\mathrm{g}$ for 15 minutes at $4^{\circ} \mathrm{C}$. Aliquots of $500 \mu \mathrm{L}$ of serum were frozen and stocked at $-80^{\circ} \mathrm{C}$ until required for assay.

The saliva samples were collected immediately after the blood collection through the Salivates Collection System (SCS) (Greiner Bio-One $\mathrm{GmbH}$, Kremsmuenter, Austria) in agreement with the manufacturer's instructions. The oral rinsing solution supplied in the SCS kit was used before the saliva collection. Subsequently, the volunteers remained with the saliva extraction solution in their mouth for two minutes. The collection beaker was opened and filled with the solution from the mouth. The saliva extraction solution was transferred into two graduate tubes via vacuum and centrifuged at $2200 \mathrm{~g}$ for 15 minutes at $4^{\circ} \mathrm{C}$. The graduated tubes measure the exact volume of collected saliva (27). The saliva quantity was determined photometrically from the extracted saliva solution at $450 \mathrm{~nm}$.

The internal standard in the saliva sample was determined by means of saliva calibrators, and the saliva quantity was then recalculated.

\section{Serum and saliva uric acid concentration}

Serum and saliva uric acid concentration was determined by automated equipment (Autolab 800) via an appropriate kit (Uricostat, Wierner lab., Argentina).

\section{Procedure for voltammetric measurements}

The voltammetric measurements were carried out with a PGSTAT-10 potentiostat from Autolab Echo Chemie (Utrecht, Netherlands) interfaced to a PC (software GPES 4.9). An electrochemical cell with three electrodes was used with a saturated calomel electrode as the reference electrode, a platinum wire as the auxiliary electrode and a glassy carbon electrode (3-mm diameter) as the working electrode. The measurements were performed under thermostatic control at $20.0 \pm 0.5^{\circ} \mathrm{C}$.

For the voltammetric measurements, $120 \mu \mathrm{L}$ of serum or saliva were used. The samples were diluted in $480 \mu \mathrm{L}$ of PBS solution (aqueous phosphate buffer $0.5 \mathrm{molL}^{-1}, 0.9 \%$ of $\mathrm{NaCl}, \mathrm{pH} 7.4$ ). The glassy carbon electrode was polished with $0.3 \mu \mathrm{m}$ alumina, rinsed thoroughly with deionized water and sonicated immediately before each experimental series in deionized water. The potential for voltammetric experiments was recorded from $-300 \mathrm{mV}$ to $+1000 \mathrm{mV}$.

\section{Statistical analysis}

The software Origin 6.0 was used for the statistical analysis of results. The results were expressed as mean \pm standard deviation. The Student t-test for paired samples was used to compare the results of CPC addition on voltammetric measurements of serum and saliva samples. Statistical significance was set at $p<0.01$. The boxplot graphs were used for a descriptive analysis of results. The graphs were made by the Matlab $^{\oplus} 7.0$ program. 

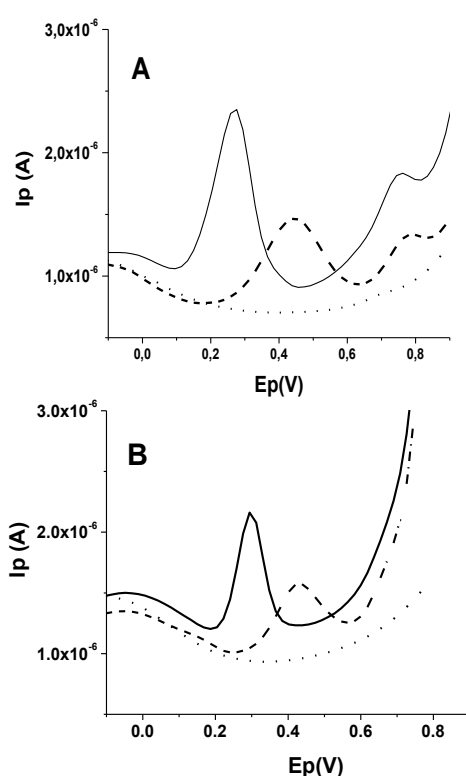

Figure 1: DPV of (A) serum and (B) saliva (diluted in PBS 1:4) in the presence of CPC $\left(100 \mathrm{mmol} \mathrm{L}^{-1}\right)$ (solid line), and absence of CPC (dashed line), PBS+CPC (dotted line)

\section{Results and Discussion}

\section{Effects of CPC on biological fluid voltammograms}

Figure 1 shows the differential pulse voltammograms obtained for the same serum (A) and saliva (B) samples in the presence and in the absence of $\mathrm{CPC}$ in a phosphate buffer solution at $\mathrm{pH}$ 7.4.

The presence of $100 \mathrm{mmolL}^{-1} \mathrm{CPC}$ (solid lines) in serum and saliva samples shifted the first peak potential (Ep) toward lower positive values. The value of the second peak potential in the serum remained unaltered. As can be seen, in presence of CPC, two oxidation potentials were observed at $0.272 \mathrm{~V}$ and $0.755 \mathrm{~V}$ (Figure 1A, solid line). Only one oxidation potential at $0.295 \mathrm{~V}$ was observed in the saliva sample voltammogram (Figure 1B, solid line). A significant enhancement of serum and saliva peak current (Ip) was verified in voltammograms (solid lines) upon surfactant addition.

The voltammograms obtained in the absence of CPC (dashed lines) were broad and showed more positive values of peak potential (Ep) than did the same sample in the presence of CPC. The oxidation potential of saliva sample voltammogram was observed at $0.413 \mathrm{~V}$ (Figure 2B, dashed line). The oxidation potentials of serum sample voltammogram occur at $0.444 \mathrm{~V}$ and $0.750 \mathrm{~V}$ (Figure 2A, dashed line). These results are similar to those obtained by cyclic voltammograms (CV) of plasma samples observed by Chevion and Kohen $[8,10]$. Literature presents the urate and ascorbate identified for HPLC-ED as the major components of the first anodic current in plasma cyclic voltammograms $[8,28,29]$.The second peak observed in plasma cyclic voltammograms can be caused mainly by NADPH, which originates from red blood cell hemolysis or lipoic acid $[10,29,30]$.

The surfactant addition shifts the oxidation peak potential and enhances the peak current as a consequence of surfactant film formation at the electrode/solution interface. The positively charged surfactant, $\mathrm{CPC}$, establishes an electrostatic interaction with two specific anionic components of the first anodic peak current, ascorbate and urate, which are negatively charged. The presence of these compounds in the first peak currents of serum and saliva voltammograms explains the increased speed of oxidation and the consequent increase in the peak current [31].

\section{Effect of CPC concentrations on biological fluids voltammograms}

The influence of CPC concentration on the peak potential (Ep) and peak current (Ip) were investigated in order to define the most effective surfactant concentration to use. Solutions containing different amounts of CPC were prepared and tested for the same serum sample. Twenty subsequent voltammograms were carried out for each CPC concentration. Figure 2 presents the data of the peak potential, Ep (A), and peak current, Ip (B), of a serum sample diluted in PBS with the surfactant concentrations. The values of variation coefficients (\%) after 20 measurements for each concentration of CPC are also presented in figure 1 beside the data points, which correspond to average values.

The Ep values decreased while Ip values increased for all CPC concentrations added, when compared with the same sample in absence of CPC. Ep and Ip variation coefficients after 20 scans in the absence of CPC were $6.9 \%$ and $29.8 \%$, respectively. The results indicate that the responses with the highest stabilization effect were obtained using $100 \mathrm{mmolL}^{-1}$ of CPC, which shows variation coefficients of $3.8 \%$ for Ep and 9.3\% for Ip. This CPC concentration provided effective stability and led to an increase and better definition of peak current. The CPC concentration of $100 \mathrm{mmolL}^{-1} \mathrm{can}$ be applied to saliva samples given that the total protein concentration is low in these samples. The saturated adsorption process of surfactants on solid surfaces generally coincides with critical micellar concentration (CMC) of surfactant [32]. The surfactants form micelles above CMC, and the solute-micelle interaction prevents the adsorption of compounds on the electrode surface. CMCs are difficult to measure with great accuracy; thus, the values found in the literature occasionally present variations [33].

$\mathrm{CPC}$ concentrations used in experiments presented in figure 1 were above CMC. CPC concentrations below CMC also were tested. The
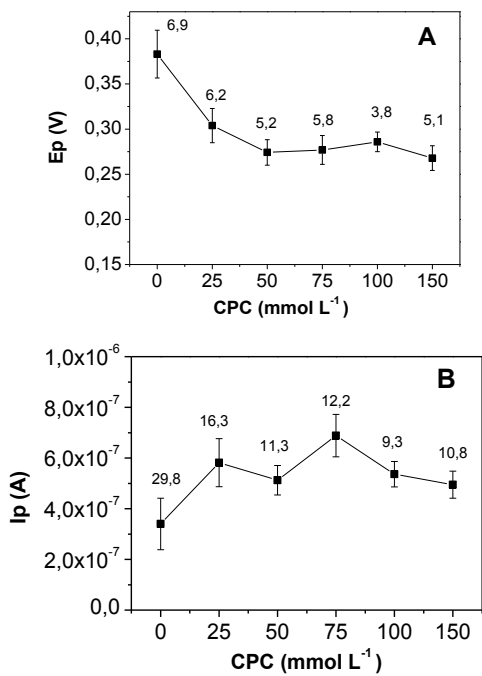

Figure 2: Variation of the peak potential $\mathrm{Ep}(\mathrm{A})$ and peak current $\mathrm{Ip}(\mathrm{B})$ in serum (diluted in PBS 1:4) with surfactant concentrations. ( $\mathrm{pH} 7.4)$. The values are mean \pm SD of 20 voltammograms for each CPC concentration. The numbers indicate the variation coefficients (\%) after 20 measurements for each concentration. 

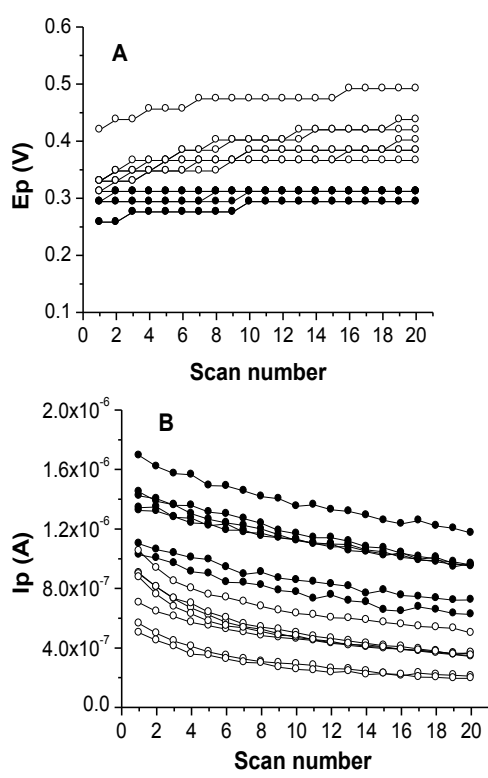

Figure 3: Ep (A) and Ip (B) of seven serum samples and Ep (C) and Ip (D) of seven saliva samples (diluted in PBS 1:4). Each line represents 20 scans series to a same sample in the absence (o) and in the presence $(\cdot)$ of $100 \mathrm{mmol}$ L-1 CPC. Significant difference $(\mathrm{p}<0.01), \mathrm{pH} 7.4$.

results were not effective, likely due to the low surfactant concentrations, which did not allow for micellar aggregate formation and the necessary solute-micelle interaction (data not shown).

Therefore, the concentration $100 \mathrm{mmolL}^{-1}$ was used for all of the results obtained in the presence of CPC.

The repeatability of the results was verified. Seven serum and saliva samples were analyzed in the presence and in the absence of CPC. For each sample, 20 voltammograms were carried out. Figure 3 presents Ep and Ip values obtained for each analyzed measure in serum (A-B) and saliva (C-D).

For all serum and saliva analysis voltammograms, the Ep values shifted toward lower positive potential values in the presence of CPC $(\mathrm{p}<0.01)$, and the results show better repeatability and stability between the first and the twentieth voltammogram. In addition, a significant increase in and better definition of peak current (Ip) were obtained in the presence of CPC.

Serum and saliva voltammograms analyzed in the absence of CPC presented broader and significantly lower peak currents $(\mathrm{p}<0.01)$, as well as low repeatability. The drop in signal during repeated DPV scans was reduced by CPC addition.

The voltammograms of a specific saliva sample presented significantly lower Ip values when compared with others saliva samples. Only 10 subsequent voltammograms of this sample were carried out in the absence of CPC due to the low concentration of analyzed compounds and the decrease in voltammetric signal, followed by complete loss of the signal during repeated scans (Figure 4). Twenty subsequent measures of this sample could be accomplished in the presence of CPC due to the increase in the peak current and larger stability of voltammetric signal.

Figure 4 shows the first and twentieth saliva sample voltammograms in the presence and in the absence of CPC.
On saliva voltammograms in the presence of $\mathrm{CPC}$, a small decrease in peak current, Ip, was found after 20 repeated voltammograms without polishing of the electrode (solid line). No differences were seen in peak potential, Ep.

In the absence of surfactant, a decrease in peak current, Ip, and a displacement in peak potential, Ep, toward larger positive values were observed after 20 repeated scans (dashed lines).

The CPC addition produced better repeatability of the measurements. The relative standard deviation for Ip values obtained after 20 subsequent voltammograms in the presence of CPC was $0.514 \times 10^{-7} \mathrm{~A}$. Ep values did not vary. In the absence of surfactant, the standard deviations were $1.172 \times 10^{7} \mathrm{~A}$ to Ip and $0.008 \mathrm{~V}$ for Ep.

The stability of a thawed sample was verified. Three aliquots of one sample were thawed at the same time. The samples were analyzed three times: immediately ( $1 \mathrm{~min}), 40$ minutes and 80 minutes after thawing. During that period, the samples remained exposed to light at room temperature $\left(20^{\circ} \mathrm{C}\right)$ in order to evaluate possible oxidation of compounds after thawing.

This same experiment was repeated four times without electrode polishing between the measurements. The objective was to verify the repeatability and to identify possible electrode passivation due to the same successive scans with electrode without polishing. A single polish was performed before Experiment no. 1. The data of Ep, Ip and area for the four experiments are presented in the figures $5 \mathrm{~A}-5 \mathrm{C}$, respectively.

The independent analysis results of each experiment show that peak potential values, Ep, were not displaced due to thawing time of samples (Figure 5A). However, peak current values, Ip, (Figure 5B) and Area (Figure 5C) can be influenced by the time of thawing, presenting a slight decrease of values because of the oxidation of compounds.

The data presented good repeatability for measurements accomplished immediately after thawing and 40 minutes after thawing. The results indicate that the decrease of voltammetric signal, in this case, can occur mainly due to oxidation of compounds during thawing. The large number of performed measurements did not result in fouling or passivation of electrode surface by adsorption of constituents from the samples.

The higher variability of the voltammogram data from Experiment no. 1 may have occurred due to the initial electrode polishing performed before beginning the experimental series. These data reinforce the interference of electrode polishing on the measurements. To minimize that initial effect, we suggested performing a larger number of scans

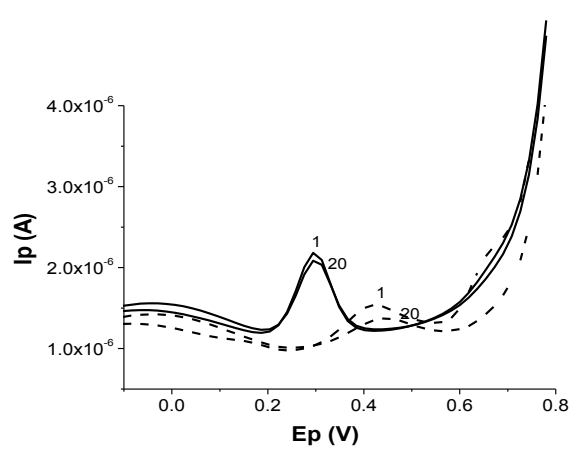

Figure 4: First and twentieth voltammogram of the same saliva sample in the presence of $100 \mathrm{mmol} \mathrm{L}^{-1} \mathrm{CPC}$ (solid line) and in the absence of CPC (dashed line). Saliva diluted in PBS 1:4 ( $\mathrm{pH} 7.4)$. 

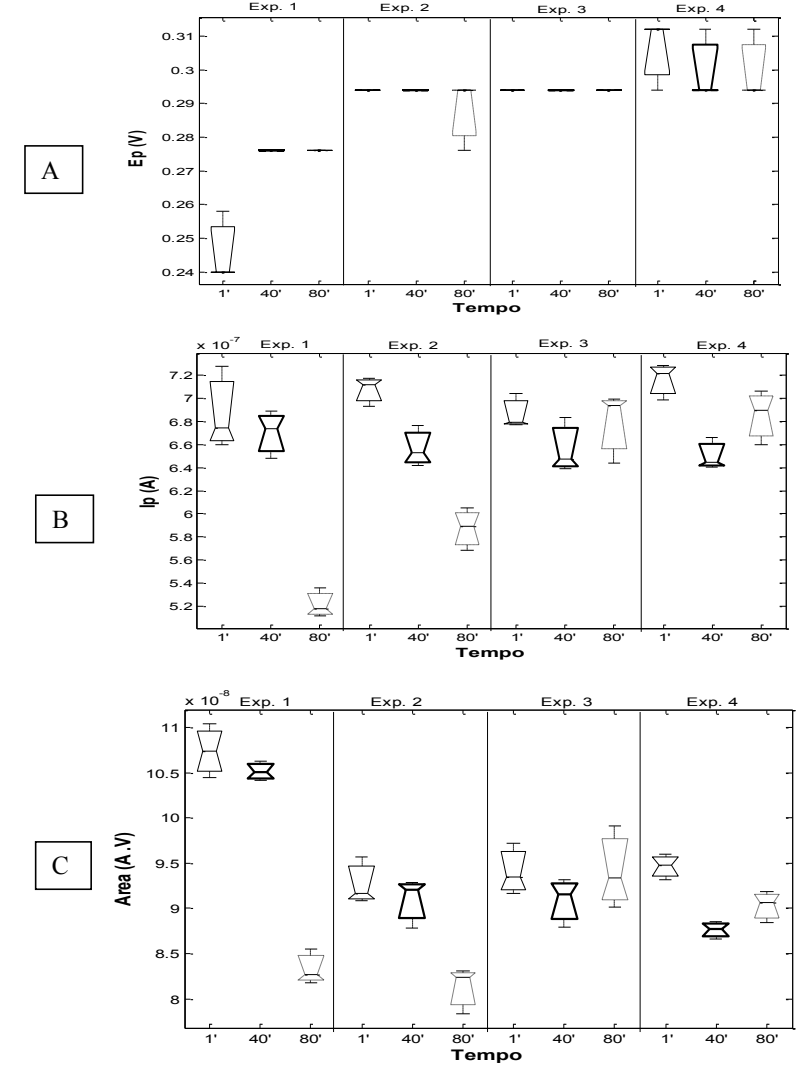

Figure 5: (A) Ep values of DPV for the same serum sample (diluted in PBS 1:4) obtained in four subsequent experiments in the presence of CPC (100

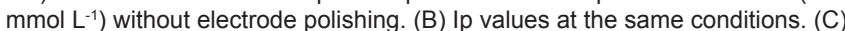
Area values at the same conditions. In each experiment, the samples were analyzed every 1, 40 and 80 minutes after thawing. All measures were carried out in triplicate.

with the buffer solution in order to stabilize the system before beginning the analysis.

\section{Effects of ascorbate and urate additions on DPV of serum} samples in the presence and absence of CPC

The sensitivity of this method was verified through ascorbate additions $(\mathrm{A}$ and $\mathrm{B})$ and urate additions $(\mathrm{C}$ and $\mathrm{D})$ to the serum. The measurements were carried out in the presence $(A, C)$ and absence $(B$, D) of CPC surfactant. The data are presented in figure 6.

In the presence of surfactant, the increasing ascorbate concentrations did not alter the peak potential value of $0.276 \mathrm{~V}$, but the peak current value decreased to $0.28 \times 10^{-6} \mathrm{~A}$ (Figure $6 \mathrm{~A}$ ). However, a slight increase of peak area to $0.052 \times 10^{-6} \mathrm{AV}$ was observed. The successive additions of urate in the presence of CPC (Figure 6C) induced the opposite effect on peak current as compared to ascorbate. The peak potential was shifted to lower positive values, and a dose-dependent increase in peak current was verified.

In the absence of the surfactant, the ascorbate additions (Figure 6B) shifted the peak potential from $0.366 \mathrm{~V}$ to $0.402 \mathrm{~V}$. The peak current decreased to $0.41 \times 10^{-6} \mathrm{~A}$, and the peak area decreased to $0.031 \times 10^{-6}$ AV. The results of urate additions were similar, with a decrease of the voltammetric signal (Figure 6D). The results indicate that in the absence of CPC, the electrode was not sensitive in detecting increases of ascorbate and urate concentrations in serum samples.
The decrease of peak current observed with ascorbate addition in the presence of CPC (Figure 6A) does not necessarily indicate a low sensitivity of the method; nor is the loss of voltammetric signal associated with fouling on electrode surface or electrode passivation. Vladmirova and Ramenskai [34] showed that CPC interacts with ascorbate and ionic associations of different compositions are formed that can alter the mechanism of ascorbate oxidation. When the ascorbate concentration exceeds the capacity to form ionic associations with CPC, the signal becomes broad, probably due to two mechanisms simultaneously competing for ascorbate oxidation.

\section{Effects of urate additions in blood serum in the presence of ascorbate}

The urate and ascorbate interaction was investigated through urate additions in serum sample containing ascorbate $\left(100 \mathrm{mmolL}^{-1}\right)$ in the presence and in the absence of CPC. The obtained data are presented in figure 7.

In the presence of $\mathrm{CPC}$, the urate additions induced a displacement of Ep to lower positive values (from $0.276 \mathrm{~V}$ for $0.150 \mathrm{~V}$ ) with dosedependent increases in peak current. Peak current and peak area values increased in response to the antioxidant concentration increase. In the absence of CPC, this effect was not observed. The peak potential showed a slight displacement from $0.40 \mathrm{~V}$ to $0.42 \mathrm{~V}$. Tendencies of peak current and peak area to decrease were observed. In the absence of $\mathrm{CPC}$, no sensitivity was observed in response to the increase in urate concentration.

These data reinforce that the first potential peak can be attributed mostly to urate and ascorbate [8]. The DPV method in the presence
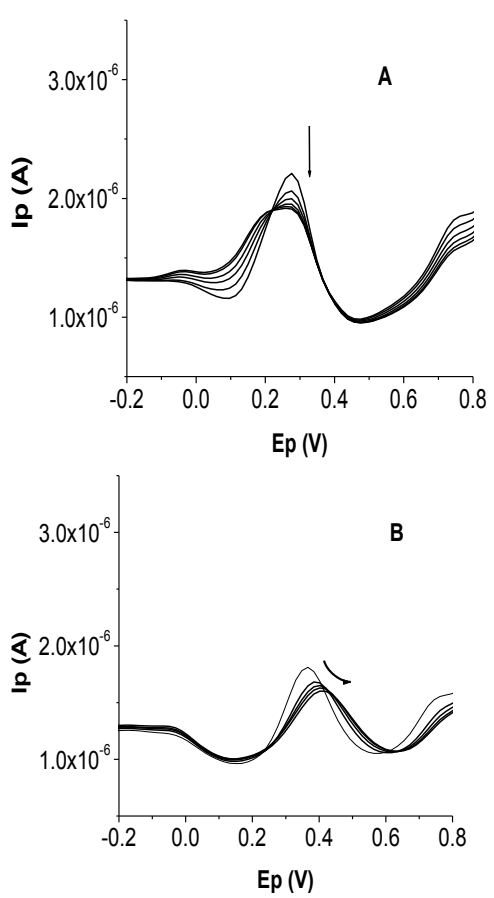

Figure 6: Ascorbate additions (concentrations: 40, 60, 80 and $100 \mathrm{mmol} \mathrm{L}^{-1}$ ) in the presence of (A) $100 \mathrm{mmol} \mathrm{L}^{-1} \mathrm{CPC}$ and in the (B) absence of CPC. Urate additions (concentrations $40,60,80$ and $100 \mathrm{mmol} \mathrm{L}^{-1}$ ) in the presence of 100 mmol L-1 CPC (C) and in the absence of CPC (D). Serum is diluted in PBS 1:4, $\mathrm{pH}$ 7.4. The arrows indicate the voltammogram behaviors with ascorbate and urate concentration increases. 

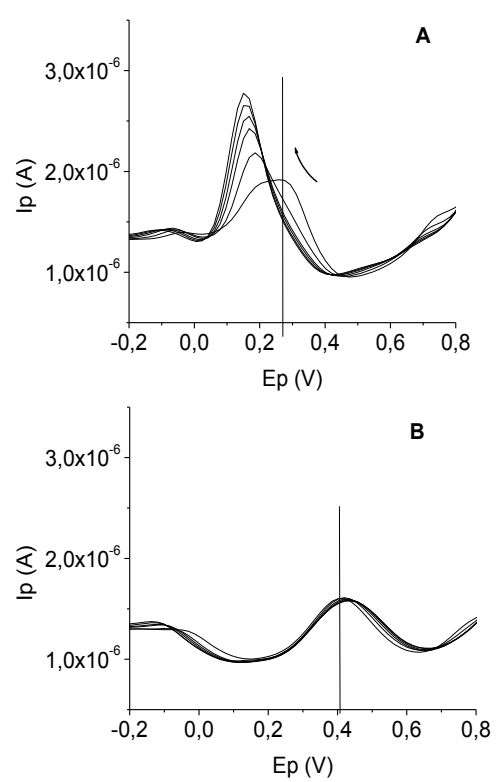

Figure 7: Urate additions (concentrations: 20, 40,60, 80 and $100 \mathrm{mmol}$ $\mathrm{L}^{-1}$ ) in serum (diluted in PBS 1:4) in the presence of $100 \mathrm{mmol} \mathrm{L}^{-1} \mathrm{CPC}(\mathrm{A})$, and in the absence of $\mathrm{CPC}(\mathrm{B}), \mathrm{pH}$ 7.4. Both samples contained ascorbate in the concentration of $100 \mathrm{mmol} \mathrm{L}^{-1}$, added previously. The measures were made in triplicate for each concentration. The arrow indicates the trend of the voltammogram behavior with urate concentration increase.
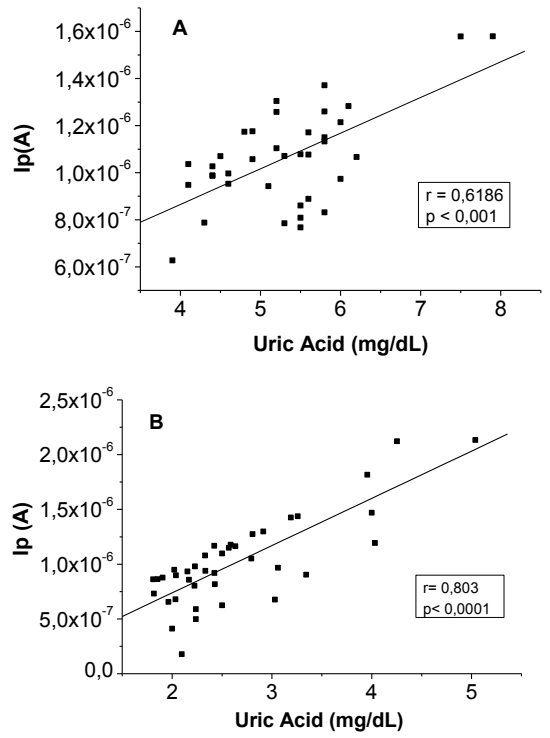

Figure 8: Correlation of peak current values (Ip) in (A) serum and (B) saliva with uric acid concentration. Serum and saliva were diluted in PBS (1:4).

of CPC was sensitive to detect additions of urate and/or ascorbate added in the serum sample, and urate negates the ascorbate effect on voltammograms.

\section{Correlations between DPV and uric acid in serum and saliva samples}

Figure 8 presents the correlation data between peak current values (Ip) and uric acid concentration values of 40 serum (A) and saliva (B) samples of young soccer players. The peak current values presented good correlation with uric acid concentration in serum $(r=0.6186$, $\mathrm{p}<0.001)$ and saliva $(\mathrm{r}=0.803, \mathrm{p}<0.0001)$ ( $\mathrm{A}$ and $\mathrm{B}$, respectively). The peak current values of serum and saliva did not present a correlation amongst themselves (data not shown). The correlation data between Ip and urate points to urate as being the main determinant of antioxidant capacity, mainly in saliva.

\section{Conclusions}

The results presented in this work demonstrate that the DPV method combined with the cationic micellar system of CPC can be considered a direct measure of the antioxidant because the results are based only on the physico-chemical properties of the antioxidant compounds, without the use of reactive species. The urate and the ascorbate are the main compounds of the first peak current.

Due to the complexity of biological samples, the separation of each antioxidant compound and individual study of their actions can be inefficient. The results would not take into account the synergistic interaction between the compounds, which is a very relevant fact when the antioxidant capacity is analyzed in a system. Moreover, the separation processes usually increase the costs.

The surfactant CPC showed highly effective results in terms of stability, sensitivity, selectivity and repeatability of antioxidant detection in serum and saliva. It can substitute for electrode polishing and prevent the electrode passivation and the drop in voltammetric signal. At the evaluated conditions, DPV is a simple method of application. The analysis time is brief, and the cost becomes lower because materials can be reused. The data presented in this study validate DPV as a methodology for serum and saliva redox state evaluation at different physiological or pathological situations.

The determination of reducing potential of biological fluids, such as saliva, can be more reliable because the samples are not influenced by diet or other interferences. The literature does not present studies of antioxidant capacity in saliva using the DPV method. The possibility of determination of antioxidant capacity through saliva is presented as a practical and non-invasive alternative.

\section{Acknowledgments}

The authors acknowledge the Brazilian Foundation FAPESP (10/02791-7) for financial support.

\section{References}

1. Blasco AJ, Crevillén AG, González MC, Escarpa A (2007) Direct Electrochemica Sensing and Detection of Natural Antioxidants and Antioxidant Capacity in Vitro Systems. Electroanalysis 19: 2275-2286.

2. Atsumi T, Iwakura I, Kashiwagi Y, Fujisawa S, Ueha T (1999) Free radical scavenging activity in the nonenzymatic fraction of human saliva: a simple DPPH assay showing the effect of physical exercise. Antioxid Redox Signal 1: $537-546$.

3. Korotkova El, Karbainov YA, Avramchik OA (2003) Investigation of antioxidant and catalytic properties of some biologically active substances by voltammetry. Anal Bioanal Chem 375: 465-468.

4. Korotkova El, Karbainov YA, Shevchuck AV (2002) Study of antioxidant properties by voltammetry. J Electroanal Chem 518: 56-60.

5. Alves AA, Pereira da Silva L, Macedo DV, Kubota LT (2003) Amperometric sensor for glutathione reductase activity determination in erythrocyte hemolysate. Anal Biochem 323: 33-38.

6. Cao G, Prior RL (1998) Comparison of different analytical methods for assessing total antioxidant capacity of human serum. Clin Chem 44: 1309-1315. 
Citation: de Passos MA, de Macedo DV, de Cássia Silva Luz R, Nunes LAS, Kubota4 LT, et al. (2012) Micellar Effects of Cetyl Pyridinium Chloride on Antioxidant Capacity, Voltammetric Response of Serum and Saliva Samples. J Biosens Bioelectron 3:130. doi:10.4172/2155-6210.1000130

Page 7 of 7

7. Halliwell B (1990) How to characterize a biological antioxidant. Free Radic Res Commun 9: 1-32.

8. Chevion S, Berry EM, Kitrossky N, Kohen R (1997) Evaluation of plasma low molecular weight antioxidant capacity by cyclic voltammetry. Free Radic Bio Med 22: 411-421.

9. Halliwell B, Gutteridge JMC (2007) Free Radicals in Biology and Medicine (3rdedn), Lavoisier Librairie.

10. Kohen R, Vellaichamy E, Hrbac J, Gati I, Tirosh O (2000) Quantification of the overall reactive oxygen species scavenging capacity of biological fluids and tissues. Free Radic Biol Med 28: 871-879.

11. Skoog DA, Holler FJ, Niemen TA (2006) PrincÃpios de AnÃ jlise Instrumental, 5a EdiÃßÃ£o, Ed. Bookman, Porto Alegre.

12. Kissinger PT, Ridgway TH (1996) Laboratory Techniques in Eletroanalytical Chemistry, M. Dekker, N. York.

13. Currell K, Jeukendrup AE (2008) Validity, reliability and sensitivity of measures of sporting performance. Sports Med 38: 297-316.

14. Atkinson G, Nevill AM (1998) Statistical methods for assessing measurement error (reliability) in variables relevant to sports medicine. Sports Med 26: 217 238

15. Hopkins WG (2000) Measures of reliability in sports medicine and science. Sports Med 30: 1-15.

16. Hoyer B, Jensen N (2006) Stabilization of the voltammetric serotonin signal by surfactants. Electrochem Commun 8: 323-328.

17. Raj CR, Tokuda K, Ohsaka T (2001) Electroanalytical applications of cationic self-assembled monolayers: square-wave voltammetric determination of dopamine and ascorbate. Bioelectrochemistry 53: 183-191.

18. Hoyer B, Jensen N (2007) Stabilization of the voltammetric response of organic analytes with self-passivating electrode reactions: Synergistic effect of surfactants and high buffer strength. J Electroanal Chem 601: 153-160.

19. Barek J, Fogg AG, Muck A, Zima J (2001) Polarography and Voltammetry at Mercury Electrodes. Critical Reviews in Analytical Chemistry 31: 291-309.

20. Hoyer B, Jensen N, Busch LP (2001) Effect of the pretreatment of recast nafion membranes on their rejection of the albumin interference in anodic stripping voltammetry. Electroanalysis 13: 843-848.
21. Banks CE, Compton RG (2003) Ultrasonically enhanced voltammetric analysis and applications: an overview. Eletroanalysis 15: 329-346.

22. Hoyer B, Jensen N (1995) Phase-inversion cellulose acetate membranes fo suppression of protein interferences in anodic stripping voltammetry. Talanta 42: 767-773.

23. Rusling JF (1991) Controlling electrochemical catalysis with surfactant microstructures. Acc Chem Res 24: 75-81.

24. Sivagnanam U, Palaniandavar M (1996) Electrochemical behaviour of certain biomimetic copper (II) complexes in aqueous and aqueous micellar solutions. $J$ Electroanal Chem 410: 43-53

25. Rusling JF (1998) Enzyme bioelectrochemistry in cast biomembrane-like films Acc Chem Res 31: 363-369.

26. Szymula M, Nazkiewickz-Michalek J (2003) Atmospheric and electrochemica oxidation of ascorbic acid in anionic, nonionic and cationic surfactant systems. Colloid Polym Sci 281: 1142-1148.

27. Raggam RB, Santner BI, Kollroser M, Gossler W, Schmied B, et al. (2008) Evaluation of a novel standardized system for collection and quantification of oral fluid. Clin Chem Lab Med 46: 287-291.

28. Chevion S, Roberts MA, Chevion M (2000) The use of cyclic voltammetry for the evaluation of antioxidant capacity. Free Radic Biol Med 28: 860-870.

29. Kohen R, Beit-Yannai E, Berry EM, Tirosh O (1999) Overall low molecular weight antioxidant activity of biological fluids and tissues by cyclic voltammetry. Methods Enzymol 300: 285-296.

30. Chevion S, Hofmann M, Ziegler R, Chevion M, Nawroth PP (1997) The antioxidant properties of thioctic acid: characterization by cyclic voltammetry Biochem Mol Biol Int 41: 317-327.

31. dos Reis AP, Tarley CR, Maniasso N, Kubota LT (2005) Exploiting micella environment for simultaneous electrochemical determination of ascorbic acid and dopamine. Talanta 67: 829-835

32. Connors TF, Rusling JF, Owlia A (1985) Determination of standard potentials and electron-transfer rates for halobiphenyls from electrocatalytic data. Anal Chem 57: 170-174.

33. le Maire M, Champeil P, Moller JV (2000) Interaction of membrane proteins and lipids with solubilizing detergents. Biochim Biophys Acta 1508: 86-111.

34. Vladimorova TV, Ramenska LM (2006) Ionic associates of ascorbic acid with cetylpyridinium chloride. Russ J Phys Chem 80: 836-837. 